\title{
LONG PERIODS IN DIAMETER, IRRADIANCE AND ACTIVITY OF THE SUN
}

\author{
Ph. Delache ${ }^{(1)}$, F. Laclare ${ }^{(2)}$, H.Sadsaoud ${ }^{(2)}$ \\ (1) Nice Observatory and CNRS (UA128) \\ B.P. 139 \\ F-06003 Nice Cedex \\ (2) C.E.R.G.A. and CNRS (UA 252) \\ Plateau de Calern, Caussols \\ F-06460 St Vallier de Thiey
}

\begin{abstract}
Long periods have been detected in solar diameter measurements obtained during the past 10 years. The same periodicities are also present in other global Sun parameters such as the $10 \mathrm{~cm}$ radio flux, the total irradiance, and possibly the neutrino flux. Special attention is paid to periods corresponding to the solar cycle ( $\left.{ }^{\circ} \mathrm{C}^{n}\right), 1000$ days $\left(" P^{n}\right)$ and 320 days $\left(" W^{n}\right)$. Studying the respective phases of the signals give some insight to the possible physical origins of these variations.
\end{abstract}

\section{PERIODS IN THE SOLAR DIAMETER}

A previous analysis of astrolabe measurements have shown the existence of at least three well identified peaks in the power spectrum (Delache et al., 1985). Figure 1a shows in a $\log$-log scale the power spectrum of ten years of observations. One observes a constant level noise spectrum whose low frequency portion contains some signal one order of magnitude larger. For comparison, figure $1 \mathrm{~b}$ shows a spectrum obtained by randomising the actual data among the actual observing days. Clearly the low frequency signal is washed out.

Each of the detected periods can be found in other global solar observables. This reinforces the confidence in the quality of our observations, but does not demonstrate that the fluctuations which we observe are actually diameter variations. To be more specific, we think that the visual definition of the limb is affected by the solar activity. For example a measurement of solar apparent "oblateness" can be deduced from our observations quite comparable to Dicke \& Goldenberg first determination (1967) which is not surprising, since we do not take into account any modification of the limb position due to plages and other active regions.

It is then very useful to study the phase relations between the various parameters that have a common peak in their power spectra. We shall consider, in turn, the 11 years, 1000 days and 320 days periods.

\section{VARIATIONS WITH THE 11-YEAR CYCLE}

Quoting Gilliland (1981) :"A $3 \sigma$ correlation between the solar radius and sunspot cycle is found at 11 years .... the correlation is negative, with maximum sunspot number corresponding to minimum solar radius. The half-amplitude of the 11 year variation is $\sim 0.1$ arc sec." Our observations confirm precisely Gilliland's amplitude and phase. One can, 

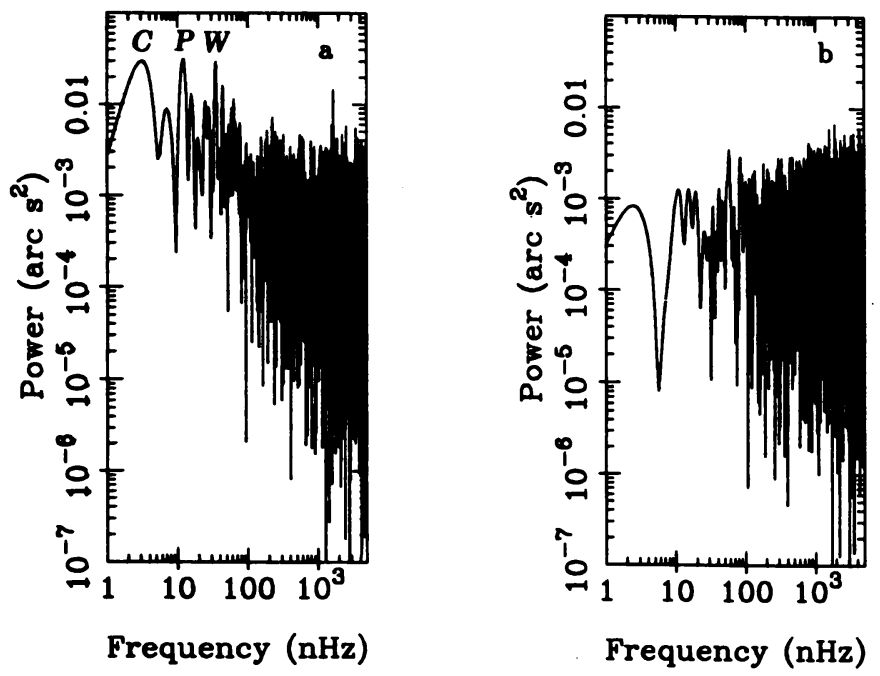

Figure 1: Power spectra of the solar diameter observations. a: original data show the main peaks, C, P, W. b: randomised data show no visible peak.

for example reconstruct signals of the diameter and of the Ottawa $10 \mathrm{~cm}$ radio flux (which measures conveniently the solar activity) filtered by a pass-band filter around (11 years $)^{-1}$. They are in perfect anticorrelation. This could already have been deduced from the negative cross-correlation between our diameter measurements and the Zürich Sunspot Number that was shown in Delache et al. (1985). One is tempted to consider this shrinking of the active Sun as real, since it agrees also with the value deduced from 5-min modes frequency shifts observed independantly by Woodard \& Noyes (1985) and by Fossat (1986).

As there seems also to be a correlation between the activity and the neutrino flux (Rowley et al., 1984), there is no surprise in the correlation between radius and neutrino flux which is shown in figure 2 . If the variations of the neutrino flux with the cycle would be real too, a possible explanation in terms of the neutrino-matter interaction is proposed by $E$. Schatzman (1986): he considers that the critical density and density gradient which are necessary to change the $e$-neutrinos into $\mu$-neutrinos (Bouchez et al. 1986) could be found at the base of the convection zone where they could vary substantially during the cycle.

\section{VARIATIONS AT 320 AND 1000 DAYS}

\subsection{In the solar activity}

Examination of Stenflo \& Vogel (1985) analysis of the solar surface magnetic fields in terms of spherical harmonics reveals a lot of power around $\mathrm{W}$ (35 $\mu H z, 320$ days), especially in the low, even parity degrees (see their figure 3). There may be also, but not entirely convincingly, some power not far from $\mathrm{C}(12 \mu \mathrm{Hz}, 1000$ days). This confirms what has already been found by Delache et al. (1985) who identified the $W$ peak in the power spectrum of Zürich Sunspot Number.

\subsection{In the solar irradiance}

Figure 3 shows a power spectrum of SMM/ACRIM irradiance data from 1980 to 1984 


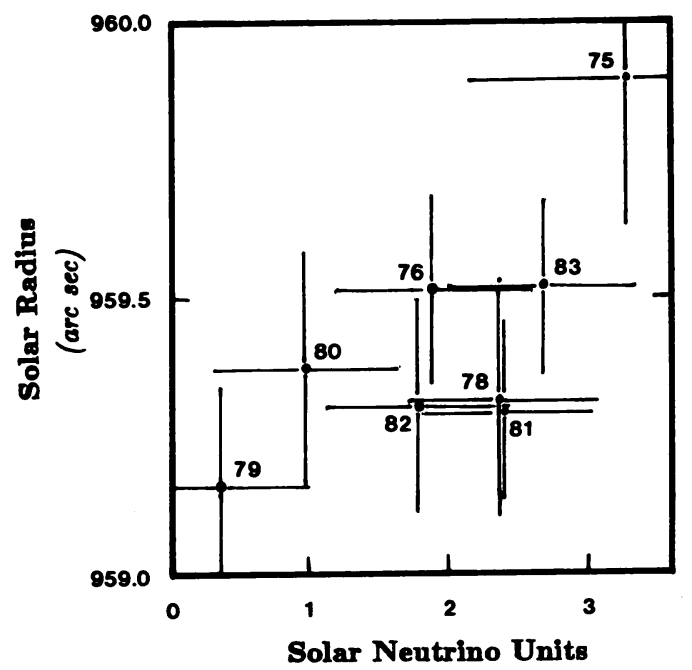

Figure 2: Solar radius (ordinate) and neutrino flux (abscissa) from 1975 to 1984. Year 1977 is missing in the radius determination. One sees a tendancy of the active Sun to be smaller and to emit less neutrinos.

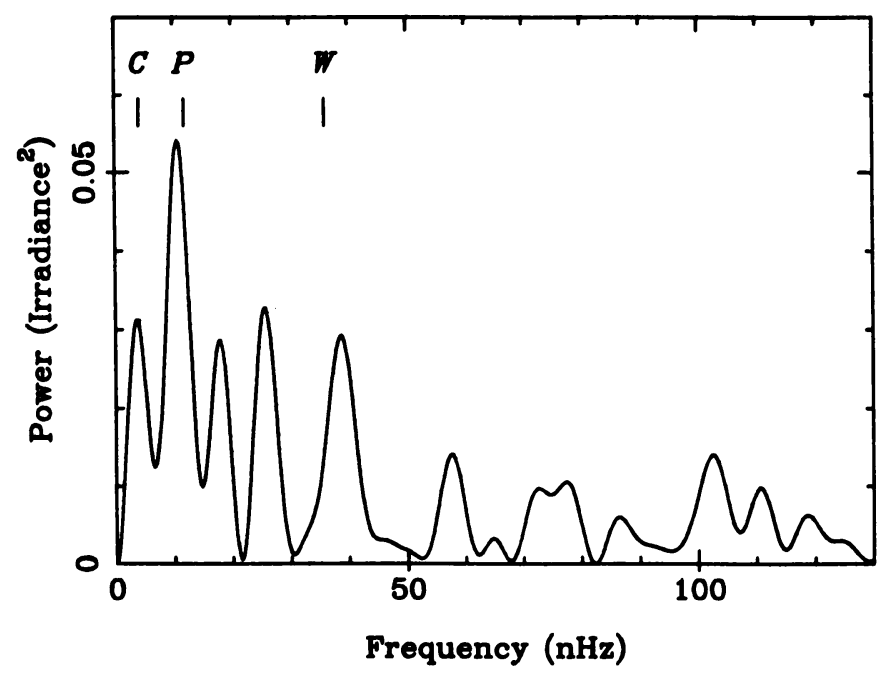

Figure 3: Power spectrum of the solar irradiance.

(courtesy R.C. Willson \& M.F. Woodard).

In order to remove the general decreasing trend of the data, a 1000 days running mean is first substracted; this eliminates the power in the lowest of the original data peaks. Of the remaining ones, it is clear from figure 3 that the $P$ peak at 1000 days dominates the spectrum, while the 320 days peak is less visible. 


\subsection{Relative phases}

We have previously shown that at 320 days, our solar diameter variations and the solar activity are in phase. Superposed epoch analysis over the available SMM/ACRIM data shows that the irradiance variations are also in phase with the other parameters at this period.

Concerning the 1000 days period, the band-pass filterings of diameter and irradiance show that they are too, exactly in phase.

\section{OTHER RECENT DIAMETER DETERMINATIONS.}

A detailed comparison is desirable with the High Altitude Observatory Solar Diameter Monitor (Brown et al. 1982) transit instrument which has been operated since 1982. One should however notice that the observed heliographic latitudes are not the same. Examination of our data shows that only the peak "P" remained over the noise level during this period of relative solar quietness.

\section{GONCLUSION}

In the case of 11 years variations we have shown why we think that the variations that we observe are really diameter variations. The situation is different for the other periods, 320 days and 1000 days.

Concerning the 320 days period, which is visible in all parameters, we consider quite possible that at this frequency, the definition of the solar limb depends on the activity, with a more active Sun appearing larger and brighter.

For the $\mathbf{P}$ peak at 1000 days which dominates the diameter and the irradiance spectra, its existence in activity indexes is only marginal. Should we, there, be measuring real diameter fluctuations the parameter $w=\frac{\delta \log R}{\delta \log L}$ is of order unity, which would correspond to " $\beta$ perturbations" located below the He ionization zone, according to Endal et al. (1985).

Among other determinations, a monitoring of solar diameter variations through the Woodard \& Noyes effect along the coming years will probably permit to infer which of our variations are due to solar activity, and which are genuine diameter fluctuations. Ground based networks and space observations of solar oscillations are also strongly needed for this purpose.

\section{REFERENCES}

Bouchez ${ }^{\top}$ et al. (1986),Z. Phys., $C$ in the press

Brown T.M. et al. (1982),Appl. Opt. 21,3588

Delache, $\mathrm{Ph}$. et al. (1985), Nature 317,416

Dicke R.H. \& Goldenberg H.M. (1967),Phys. Rev. Lett. 18,313

Endal A.S. et al. (1985),Ap.J. 290,748

Fossat E. (1986), this symposium

Gilliland R.L. (1981),Ap.J. 248,1144

Rowley J.K. et al. (1985) in Solar Neutrinos and Neutrino Astronomy, edited by Cherry M.L. et al., Am. Inst. of Phys.

Schatzman E. (1986) personal communication

Stenflo J.O. \& Vogel M. (1986), Nature 319,285

Woodard M.E. \& Noyes R.W. (1985), Nature 318,449 